Acta Crystallographica Section A

Foundations of Crystallography

ISSN 0108-7673

Received 3 August 2004

Accepted 8 October 2004

(C) 2005 International Union of Crystallography Printed in Great Britain - all rights reserved

\section{The Peierls transition in low-dimensional electronic crystals}

\author{
Sander van Smaalen
}

Laboratory of Crystallography, University of Bayreuth, 95440 Bayreuth, Germany. Correspondence e-mail:smash@uni-bayreuth.de

An overview is presented of low-dimensional electronic crystals that undergo a phase transition towards a low-temperature charge-density-wave (CDW) state. The emphasis is on inorganic compounds that develop an one-dimensional (1D) CDW. Low-dimensional features of crystal structures are discussed in relation to 1D electronic properties. X-ray diffraction is discussed as a tool to obtain quantitative information about the normal state and its structural fluctuations, and about the CDW, both in equilibrium and in its non-linear conducting state.

\section{Introduction}

Fifty years ago, Peierls (1955) showed that a one-dimensional (1D) metal is unstable against a periodic deformation of its lattice, with the period given by twice the Fermi wavevector $\left(\mathbf{k}_{\mathrm{F}}\right)$. The periodic lattice deformation (PLD) is responsible for a lowering of the energies of the filled one-electron states of the conduction band, while empty states are raised in energy (Fig. 1). The gain in electron energy is proportional to $u^{2} \ln (u)$ for small lattice distortions $u$, while the cost in strain energy is proportional to $u^{2}$, thus explaining the stability of the deformed state at a finite value of the lattice distortion.

The charge density of the conduction band exhibits a periodic variation with the same wavevector as the PLD; this is called the charge-density wave (CDW). A crystal with a Peierls distortion is said to be in a CDW state.

For a crystal with a half-filled band, the CDW state corresponds to a periodic structure with a lattice constant that is twice as large as the periodicity of the basic structure. In general, however, the filling of the conduction band is not related to the lattice periodicity of the crystal, and $\mathbf{k}_{\mathrm{F}}$ can be any fraction of the basis vector of the reciprocal lattice. In these cases, the CDW state of the crystal is characterized by an

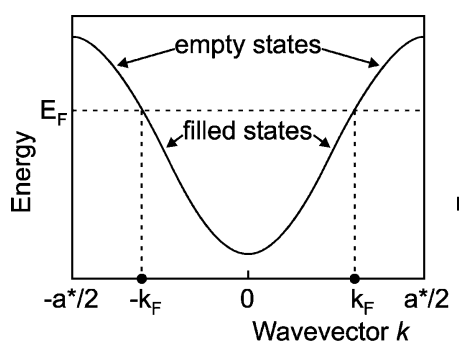

(a)

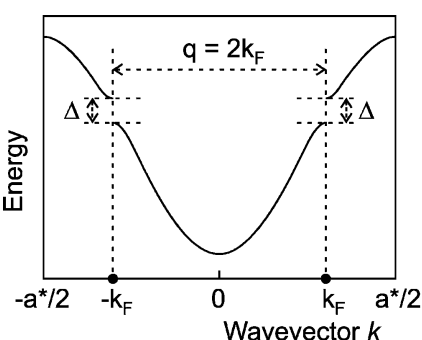

(b)
Figure 1

The conduction band of a one-dimensional crystal. One-electron states with energy $E(\mathbf{k})<E\left(\mathbf{k}_{\mathrm{F}}\right)$ are filled, while states with $E(\mathbf{k})>E\left(\mathbf{k}_{\mathrm{F}}\right)$ are empty. (a) Periodic crystal with $\pm \mathbf{a}^{*} / 2$ describing the boundary of the first Brillouin zone. (b) $1 \mathrm{D}$ crystal with a PLD with period $\mathbf{q}=2 \mathbf{k}_{\mathrm{F}}$, showing an energy gap $(\Delta)$ in the dispersion at $\mathbf{k}= \pm \mathbf{k}_{\mathrm{F}}$. incommensurately modulated structure. Incommensurate CDWs are particularly interesting because they show physical properties that differ from the properties of ordinary crystals. Most prominent is the non-linear conductivity, as has been found in several CDW compounds (Fig. 2) (Grüner, 1994).

The CDW of the conduction band and the PLD describing displacements of atoms are different aspects of a single phenomenon. Depending on the property that is studied, either the CDW or the PLD is probed. For example, scanning tunneling microscopy (STM) depends on the density of states (DOS) at the Fermi level, and thus probes the CDW directly (Magonov \& Whangbo, 1996). The electrical resistance is determined by electron-phonon coupling, and thus depends on both the CDW and the PLD. X-ray diffraction almost exclusively probes the positions of the atoms, because it does not depend on the details of the valence-electron distribution.

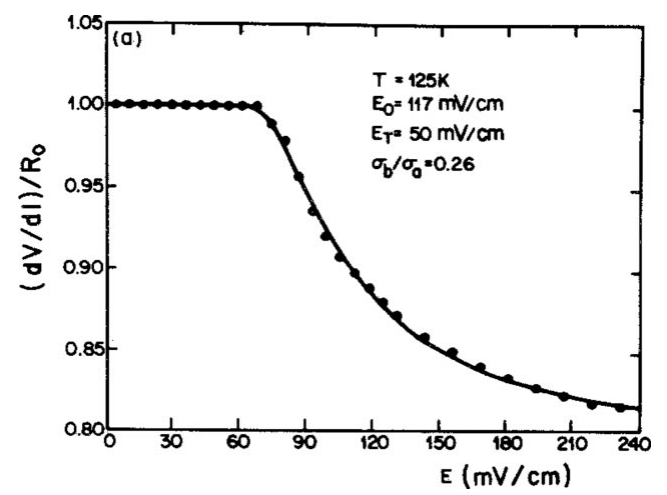

Figure 2

Non-linear electrical conductivity of $\mathrm{NbSe}_{3}$ in the CDW state. The normalized residual resistance $R / R_{0}=\left(1 / R_{0}\right) \mathrm{d} V / \mathrm{d} I(V=$ voltage, $I=$ current, $R_{0}$ is value of $R$ at $V=0$ ) is plotted as a function of the electrical field $(E)$. Ohm's law is expressed by $R / R_{0}=1$ for low fields. Above a certain threshold field $\left(E_{0}\right)$, incommensurate CDW crystals have a second conduction path due to sliding of the CDW that results in a resistance smaller than that given by Ohm's law $\left(R / R_{0}<1\right)$. Reprinted figure with permission from Fleming (1980). Phys. Rev. B, 22, 5606-5612. Copyright (1980) by the American Physical Society. 
The periodicity $\mathbf{q}$ of the PLD is responsible for the occurrence of additional Bragg reflections in the X-ray diffraction (satellite reflections) at positions $\pm \mathbf{q}$ around each Bragg reflection of the underlying basic structure. In the case of pure $1 \mathrm{D}$ crystals, $\mathbf{q}=2 \mathbf{k}_{\mathrm{F}}$.

The present contribution aims at giving an overview of compounds with a 1D electronic structure. It is discussed how $1 \mathrm{D}$ electronic systems can occur in 3D crystals $(\$ 2)$. Properties of the CDW transition and the CDW state are discussed, concentrating on the temperature dependence of the X-ray diffraction (\$3). An overview is then presented of how electronic systems are realized in different compounds. The relation between CDW and PLD is analyzed in view of explaining the mechanism of the CDW transition (\$4). Finally, 2D electronic crystals and the competition between 1D and 2D CDWs is presented $(\$ 5)$.

\section{Electronic band structures of low-dimensional electronic crystals}

One-dimensional crystals do not exist. Materials occupy a finite volume in space, and thus are 3D by definition. However, 1D electron bands can reside in 3D crystals if the atomic orbitals comprising the conduction band show an appreciable overlap in one direction while they have much smaller overlaps in the perpendicular directions. The crystal then is composed of parallel metallic chains, with weak interchain interactions only. The metallic chains can be embedded in a matrix of non-metallic atoms, such that the crystal structure contains chemical bonds in all three directions of space. Consequently, quasi-one-dimensional (1D) electronic crystals are 3D as far as the phonons are concerned. Because the PLD is an intrinsic part of the CDW state, this resolves the seeming contradiction that phase transitions cannot occur in 1D systems: all low-dimensional electronic materials are $3 \mathrm{D}$, and the CDW state (if present) is separated from the normal state by a phase transition at a temperature $T_{\mathrm{CDW}}$.

The energy of one-electron states in a 1D crystal depends on the $1 \mathrm{D} \mathbf{k}$ vector, as expressed by the dispersion relation of the $1 \mathrm{D}$ electron band depicted in Fig. 1(a). The Fermi surface

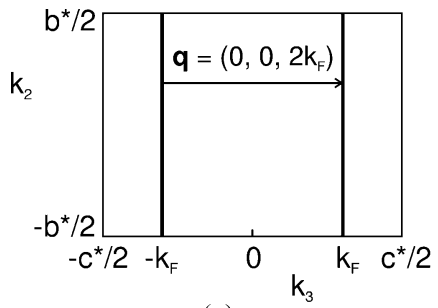

(a)

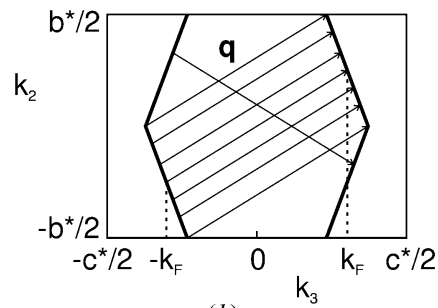

(b)
Figure 3

2D Sections of the Fermi surfaces of quasi-one-dimensional crystals. (a) Non-interacting metallic chains parallel to [001] with $\left(k_{1}, k_{2}, 0\right)$ describing $\mathbf{k}$ vectors perpendicular to the chains. (b) Weakly interacting metallic chains resulting in a warping of the Fermi surface. Perfect nesting is obtained for $\mathbf{q}=\left(0,1 / 2, q_{3}\right)$, where $q_{3}$ assumes the value such that $\mathbf{q}$ fulfills the nesting condition. Notice that the second half of the Fermi surface is nested by $\mathbf{q}^{\prime}=\mathbf{q}-\mathbf{b}^{*}$, which is equivalent to $\mathbf{q}$. is composed of the two points $\pm \mathbf{k}_{\mathrm{F}}$. The dispersion relations of the electron bands in 3D crystals are defined by the function $E_{j}(\mathbf{k})$, where $\mathbf{k}=\left(k_{1}, k_{2}, k_{3}\right)$ is a $3 \mathrm{D}$ reciprocal vector with values in the first Brillouin zone and $j$ enumerates different bands. The Fermi surface generally is a warped plane separating $\mathbf{k}$ vectors corresponding to filled and empty states, respectively.

A crystal comprising non-interacting 1D metallic chains will have a valence band with a $1 \mathrm{D}$ dispersion (Fig. 1a) for variation of the component of $\mathbf{k}$ parallel to the chains, while this band will be dispersionless for variations of the two perpendicular components of $\mathbf{k}$. The Fermi surface comprises two flat planes going through and being perpendicular to the points $\left(0,0, \pm k_{\mathrm{F}}\right)$, if [001] is the direction of the chains (Fig. $\left.3 a\right)$. The vector $\mathbf{q}=\left(0,0, q_{z}\right)$ with $q_{z}=2 k_{\mathrm{F}}$ connects every point of the plane $\left(0,0,-k_{\mathrm{F}}\right)$ of the Fermi surface with a point of the plane $\left(0,0, k_{\mathrm{F}}\right)$. This condition is called perfect nesting, and a PLD with modulation wavevector $\mathbf{q}$ will lower the energy of all oneelectron states below the Fermi surface. Consequently, the CDW state, characterized by $\mathbf{q}$, will be of lower energy than the normal state. This contrasts electronically with 3D crystals, where the nesting condition for each prospective vector $\mathbf{q}$ can be fulfilled for a single point only. The gain in electronic energy for the few states near this point then is never sufficient to overcome the cost in elastic strain energy, to which the entire crystal contributes.

In real crystals, weak interactions exist between the $1 \mathrm{D}$ metallic chains. The electron bands have a small dispersion in the directions perpendicular to the chains, while the characteristic 1D dispersion remains parallel to the chains. The Fermi surface is slightly warped, resulting in two modifications of the nesting conditions. Firstly, any given vector $\mathbf{q}$ will be a good nesting vector for only part of the Fermi surface. A gap in the one-electron states will appear for the nested fraction of the Fermi surface, while the remaining fraction will keep the compound metallic. Secondly, modulation wavevectors with non-zero components along the perpendicular directions often provide better nesting than $\left(0,0, q_{z}\right)$ (Fig. $\left.3 b\right)$. Owing to crystal symmetry, perpendicular components of $\mathbf{q}$ usually assume special values, like $1 / 2$.

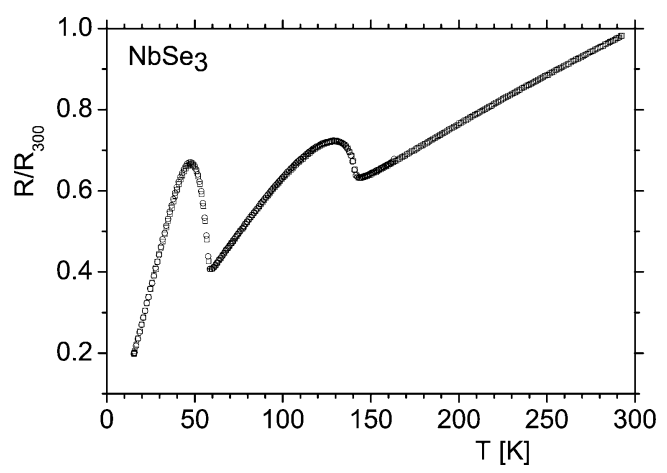

Figure 4

Temperature dependence of the electrical resistance $(R)$ of $\mathrm{NbSe}_{3}$, normalized to the value at $300 \mathrm{~K}\left(R_{300}\right)$. Data obtained on cooling (squares) and heating (circles) coincide. They reproduce the behavior published by Chaussy et al. (1976). 
Interchain interactions thus explain the metallic character of the CDW state as well as non-zero perpendicular components of the modulation wavevectors. These features are illustrated by $\mathrm{NbSe}_{3}$. $\mathrm{NbSe}_{3}$ exhibits two independent CDW phase transitions, each of which removes part of the Fermi surface as expressed by the anomalous increase of the electrical resistivity on going down through the phase transitions (Fig. 4) (Monceau, 1985). Band-structure calculations at different levels of approximation have shown that several bands cross the Fermi surface (Canadell et al., 1990). A recent calculation employing density functional theory (DFT) gives the most accurate representation of the the Fermi surface (Schafer et al., 2001). One pair of bands provides imperfect nesting conditions for $\mathbf{q}^{1}=(0,0.241,0)$, while a second pair of bands provides imperfect nesting conditions for the modulation wavevector of the second CDW, $\mathbf{q}^{2}=(1 / 2,0.263,1 / 2)$ with non-zero perpendicular components (Fig. 5).

\section{X-ray diffraction and charge-density-wave transitions}

\subsection{The fluctuating regime}

The CDW or Peierls phase transition separates the normal state at high temperatures from the CDW state at low temperatures. Macroscopically, the Peierls transition is characterized by a sudden loss of electrical conductivity on cooling through the transition, resulting in a semiconductor or in a compound with reduced metallic conductivity, depending on perfect or imperfect nesting of the Fermi surface (Figs. 4 and 5).

More detailed studies of the electronic properties of the normal states of $1 \mathrm{D}$ metals have shown that their behavior can be described within the so-called Luttinger model, which (a) Fermi Surfaces

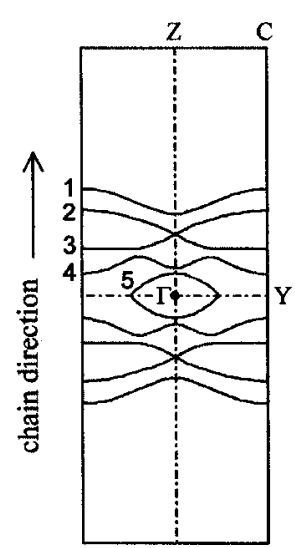

(b) Linear Nesting

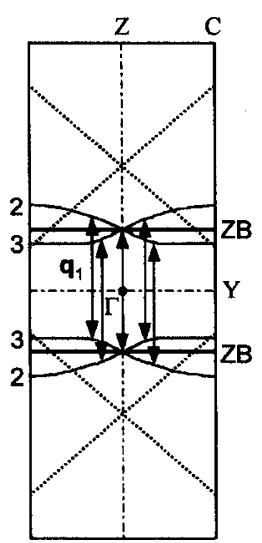

(c) Diagonal Nesting

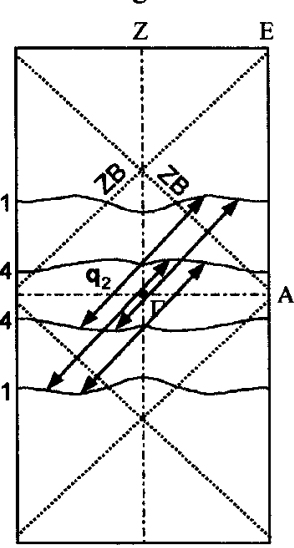

\section{Figure 5}

Sections of the Fermi surface of $\mathrm{NbSe}_{3}$, showing the nesting of bands 2 and 3 with $\mathbf{q}^{1}=(0,0.241,0)$ (responsible for the CDW at $\left.T_{\mathrm{CDW}}=145 \mathrm{~K}\right)$, and of bands 1 and 4 with $\mathbf{q}^{1}=(1 / 2,0.263,1 / 2)$ (responsible for the second $\mathrm{CDW}$ at $\left.T_{\mathrm{CDW}}=59 \mathrm{~K}\right)$. Reprinted figure with permission from Schafer et al. (2001). Phys. Rev. Lett. 87, 196403. Copyright (2001) by the American Physical Society. predicts a different behavior, e.g. of low-energy excitations, from the Fermi-liquid model of 3D metals (Voit, 1995). In recent years, spectroscopic evidence has been obtained for the Luttinger-type behavior of several 1D metals (Claessen et al., 2002).

Smaller interchain interactions make better 1D systems, and they lead to more pronounced 1D properties, like Luttingertype behavior or the development of a CDW. On the other hand, a CDW with long-range order requires 3D interactions, which can only develop due to interchain interactions. These opposite requirements on the $1 \mathrm{D}$ character result in CDW transitions occurring at temperatures $T_{\mathrm{CDW}}$ well below the transition temperatures $T_{\mathrm{MF}}$ computed on the basis of meanfield theory. Between $T_{\mathrm{CDW}}$ and $T_{\mathrm{MF}}, 1 \mathrm{D} \mathrm{CDW}$ fluctuations are present, as evidenced by planes of diffuse scattering in the X-ray diffraction (Fig. 6) (Pouget, 2004). The latter are oriented perpendicular to the 1D chains at positions $\left(0,0, \pm 2 k_{\mathrm{F}}\right)$ off the main Bragg reflections. Their widths usually increase with increasing temperature, indicating a temperature-dependent finite correlation length of the fluctuations along the chains. On approaching $T_{\mathrm{CDW}}$ from above, a crossover between a $1 \mathrm{D}$ and $3 \mathrm{D}$ fluctuating regime is usually found, which transforms into 3D long-range order at $T_{\mathrm{CDW}}$ (Pouget, 1996). Observations of diffuse scattering above $T_{\mathrm{CDW}}$ have been made for many CDW materials, including the 1D organic conductors (Pouget, 2004), $\mathrm{NbSe}_{3}$ (Pouget, Moret et al., 1983) and $\mathrm{K}_{0.3} \mathrm{MoO}_{3}$ (Pouget, Kagoshima \& Schlenker, 1983).

\subsection{The $\mathrm{CDW}$ regime}

The CDW has long-range order below $T_{\mathrm{CDW}}$. In X-ray diffraction, this is expressed by the occurrence of satellite reflections at positions $m \mathbf{q}$ ( $m$ is an integer) around the main Bragg reflections of the high-temperature lattice. For commensurate CDWs (e.g. $\left.\mathbf{q}=1 / 2 \mathbf{b}^{*}\right)$, the deformed atomic structure can be described with respect to a supercell ( $a \times 2 b \times c$ in the example), but for incommensurate CDWs

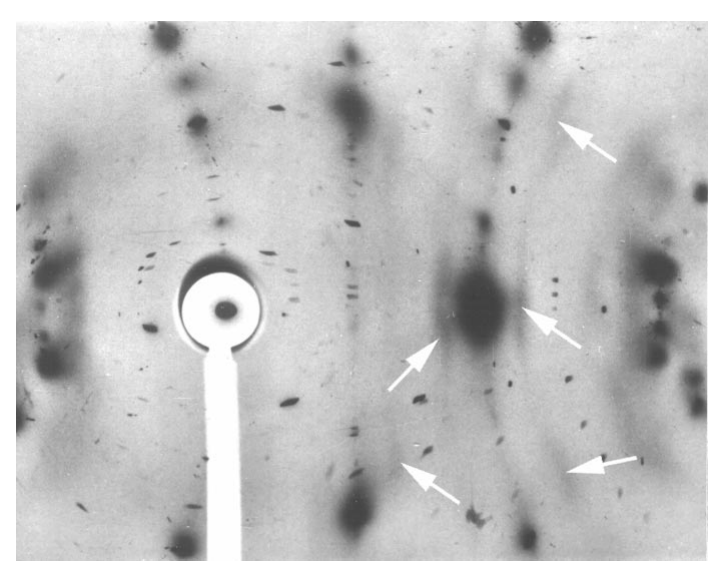

Figure 6

X-ray scattering of $\mathrm{NbSe}_{3}$ at $T=295 \mathrm{~K}$. White arrows point towards 1D diffuse scattering of the $\mathbf{q}^{1} \mathrm{CDW}$. The $\mathbf{b}$-axis has horizontal orientation. Courtesy J. P. Pouget, see Pouget (1989) and Pouget, Kagoshima \& Schlenker (1983). 
an integer indexing of all Bragg reflections is only possible with the use of four reciprocal basis vectors, according to

$$
\mathbf{H}=h \mathbf{a}^{*}+k \mathbf{b}^{*}+l \mathbf{c}^{*}+m \mathbf{q},
$$

where $\mathbf{H}$ is the scattering vector and $(h, k, l, m)$ are the integer indices of the Bragg reflections. The modulation wavevector $\mathbf{q}$ is equal to the wavevector of the PLD and is defined by components $\left(\sigma_{1}, \sigma_{2}, \sigma_{3}\right)$ with respect to the reciprocal basis vectors of the basic structure:

$$
\mathbf{q}=\sigma_{1} \mathbf{a}^{*}+\sigma_{2} \mathbf{b}^{*}+\sigma_{3} \mathbf{c}^{*}
$$

Satellite reflections $(m \neq 0)$ may have equal widths as main reflections, indicating true long-range order of the CDW (Lüdecke, Jobst \& van Smaalen, 2000). Alternatively, satellites broader than main reflections indicate a limited order of the CDW, but it may also be the result of microtwinning, when the CDW transition is accompanied by a reduction of point symmetry (Galli et al., 2002). Usually, CDW satellite reflections are weak $\left(10^{-2}-10^{-5} \times I_{\text {main }}\right)$, and they can be observed for values of $|m|$ equal to 1 or 2 only.

The Peierls transition is a second-order phase transition for incommensurate CDWs, while it becomes first order for commensurate CDWs. The amplitude of the PLD may serve as an order parameter, whose value increases on decreasing temperature. In a first approximation, the intensities of the satellite reflections are proportional to the square of the amplitude of the PLD. The second-order character of CDW phase transitions has thus been established by measuring the temperature dependence of the intensities of satellite reflections that are zero above $T_{\mathrm{CDW}}$ and that continuously increase on decreasing temperature below $T_{\mathrm{CDW}}$ (Fig. 7). For $\mathrm{K}_{0.3} \mathrm{MoO}_{3}$, the ratio of intensities of satellites has been found as $I_{m=2}: I_{m=1}: I_{\text {main }}=10^{-4}: 10^{-2}: 1$ for all temperatures below $T_{\mathrm{CDW}}$, indicating that the second-order satellites are the result of higher-harmonic scattering off the sinusoidal PLD

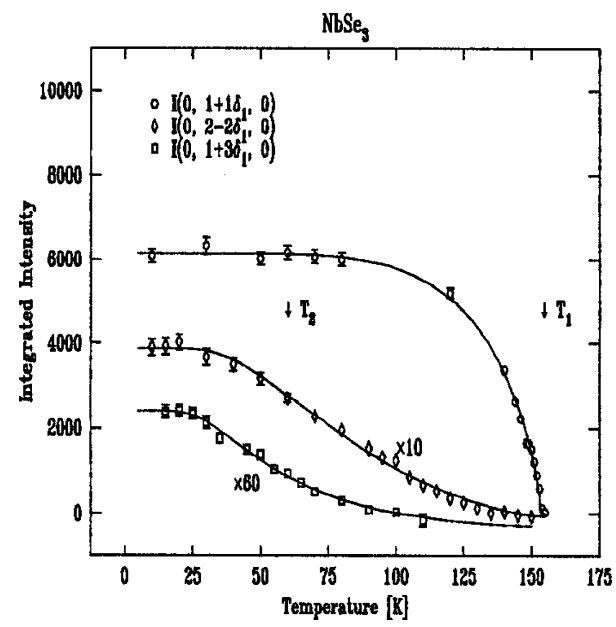

Figure 7

Intensities of satellite reflections of $\mathrm{NbSe}_{3}$ as a function of temperature. Below $T=25 \mathrm{~K}$ the second-order satellite $\left(|m|=2 ; 2 \delta_{1}\right)$ is about $16 \times$ weaker than the first-order satellite $\left(|m|=1 ; \delta_{1}\right)$. Reprinted with permission from Moudden et al. (1993). J. Phys. (Paris) IV, 3, C2, 121-124.
(Pouget et al., 1985). However, on cooling $\mathrm{NbSe}_{3}$, the intensities of the second-order satellites grow more compared to the first-order satellites (Fig. 7), suggesting the development of a secondary order parameter (second-harmonic PLD) well below $T_{\mathrm{CDW}}$ (Moudden et al., 1993). Alternatively, the firstand second-order satellites can be due to independent CDWs, as has been found in TTF TCNQ and $\mathrm{NiTa}_{2} \mathrm{Se}_{7}$ (Pouget et al., 1976; Lüdecke, Schneider \& van Smaalen, 2000).

For incommensurate CDWs, the state of the crystal is independent of the phase of the modulation. Based on this observation, Overhauser (1971) proposed that sliding of the CDW corresponds to a zero-frequency phonon with a finite wavevector equal to $\mathbf{q}$, the so-called phason mode. However, the phason mode is pinned by impurities, lattice defects and the surface, resulting in a finite frequency of this mode at all wavevectors. Pinning of the CDW can be overcome by sufficiently large electrical fields. For fields larger than a threshold field $\left(E_{T}\right)$, the sliding CDW provides a second conduction path next to single-particle electron conduction (Fleming \& Grimes, 1979). Macroscopically, this leads to non-linear electrical conductivity for large fields (Fig. 2). All conductivity experiments have been performed by attaching metallic electrodes to CDW samples. This requires that any contribution of a sliding CDW to the conductivity is to be transformed into electronic conduction near the electrodes. Theoretical descriptions explain this process in terms of an elastic deformation of the CDW, whose magnitude depends on the distance from the electrodes (for example see Brazovskii et al., 2002). Deformations of a sliding CDW have been observed by shifts of satellite reflections (i.e. changes of the magnitudes of $\mathbf{q}$ ) depending on the position between the electrodes of the portion of the sample that is illuminated by the X-ray beam (Fig. 8).

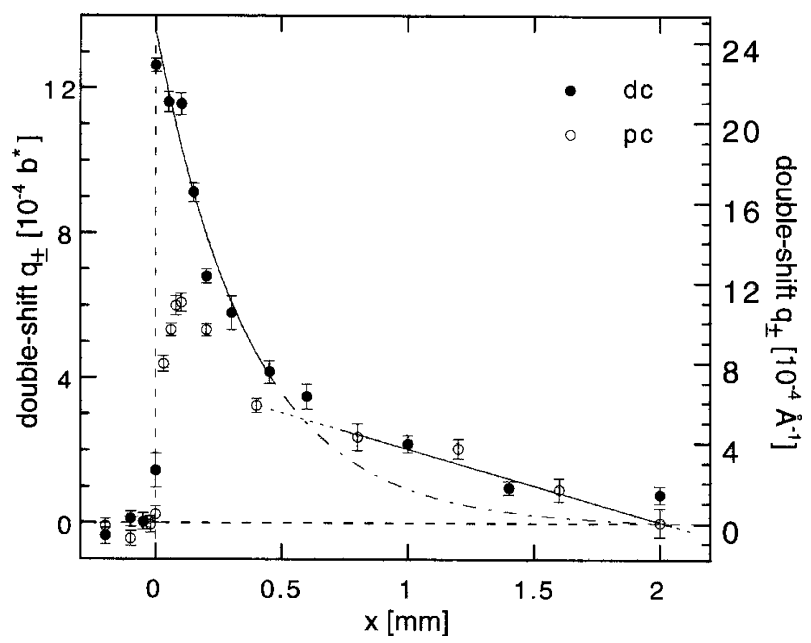

Figure 8

Deformation of the sliding $\mathrm{CDW}$ in $\mathrm{NbSe}_{3}$ as a function of the position between the electrodes (DiCarlo et al., 1993). Shown is the shift of the satellite reflection in X-ray diffraction as a function of the distance between the electrode at $x=0$ and the illuminated portion of the sample. Reprinted figure with permission from Requardt et al. (1998). Phys. Rev. Lett. 80, 5631-5634]. Copyright (1998) by the American Physical Society. 
Table 1

Inorganic compounds with a $1 \mathrm{D} \mathrm{CDW}$.

Given are the chemical formula of the compound, the space group of the basic structure, the transition temperature $\left(T_{\mathrm{CDW}}\right)$ towards the CDW state, the modulation wavevector $(\mathbf{q})$ in the CDW state and the superspace group of the CDW state. Furthermore the temperature and commensurate wavevectors $\left(\mathbf{q}_{c}\right)$ of a possible lock-in transition are given. Citations refer to publications presenting the crystal structure of the basic structure, the discovery of satellite reflections and the modulated crystal structure of the CDW state. Two lines for a single compound indicate the presence of two independent CDWs at low temperatures.

\begin{tabular}{|c|c|c|c|c|c|c|}
\hline Compound & Space group & $T_{\mathrm{CDW}}(\mathrm{K})$ & $\mathbf{q}$ & Superspace group & $T_{\text {lock-in }}(\mathrm{K})$ & $\mathbf{q}_{c}$ \\
\hline \multirow{2}{*}{${ }^{(a)} \mathrm{NbSe}_{3}$} & $P 2_{1} / m$ & 145.0 & $(0,0.243,0)$ & $P 2_{1} / m(0 \beta 0) 00$ & - & \\
\hline & & 59.0 & $(1 / 2,0.259,1 / 2)$ & $P 2_{1} / m(0 \beta 0)\left(1 / 2 \beta^{\prime} 1 / 2\right)$ & - & \\
\hline${ }^{(b)} m-\mathrm{TaS}_{3}$ & $P 2_{1} / m$ & 240.0 & $(0,0.254,0)$ & - & - & \\
\hline (b) $o-\mathrm{TaS}_{3}$ & $C 222_{1}$ & 210.0 & $(1 / 2,1 / 8,0.250)$ & - & 130.0 & \\
\hline${ }^{(c)} \mathrm{ZrTe}_{3}$ & $P 2_{1} / m$ & 63.0 & $(0.93,0,0.33)$ & - & & \\
\hline${ }^{(d)} \mathrm{FeNb}_{3} \mathrm{Se}_{10}$ & $P 2_{1} / m$ & 140 & $(0,0.26,0)$ & - & - & \\
\hline${ }^{(e)} \mathrm{NiTa}_{2} \mathrm{Se}_{7}$ & $\mathrm{C} 2 / \mathrm{m}$ & 55 & $(0,0.48,0)$ & $C 2 / m(0 \beta 0) 00$ & - & \\
\hline (h) $\left(\mathrm{NbSe}_{4}\right)_{10 / 3} \mathrm{I}$ & $P 4 / m c c$ & 285.0 & $(0,0,0.487)$ & & - & \\
\hline (j) $\left(\mathrm{TaSe}_{4}\right)_{2} \mathrm{I}$ & $I 422$ & 263.0 & $(0.05,0.05,0.085)$ & $F 211(0 \beta \gamma)$ & - & \\
\hline (j) $\left(\mathrm{NbSe}_{4}\right)_{2} \mathrm{I}$ & I422 & 210.0 & $(0.065,0.065,0.159)$ & & _- & \\
\hline${ }^{(k)} \mathrm{Nb}_{3} \mathrm{Te}_{4}$ & $P 6_{3} / m$ & 110.0 & $(1 / 3,1 / 3,3 / 7)$ & _- & _- & \\
\hline${ }^{(l)} \mathrm{In}_{0.75} \mathrm{Nb}_{3} \mathrm{Te}_{4}$ & $P 6_{3} / m$ & 137.0 & $(1 / 3,1 / 3,4 / 9)$ & - & - & \\
\hline${ }^{(l)} \mathrm{Tl}_{0.5} \mathrm{Nb}_{3} \mathrm{Te}_{4}$ & $P 6_{3} / m$ & $\sim 200$ & $(1 / 3,1 / 3,0.46)$ & - & - & \\
\hline${ }^{(q)} \mathrm{K}_{0.3} \mathrm{MoO}_{3}$ & $C 2 / m$ & 183.0 & $(0,0.748,1 / 2)$ & $C 2 / m(0 \beta 1 / 2) s 0$ & - & - \\
\hline${ }^{(q)} \mathrm{Rb}_{0.3} \mathrm{MoO}_{3}$ & $C 2 / m$ & 183.0 & $(0,0.748,1 / 2)$ & $C 2 / m(0 \beta 1 / 2) s 0$ & - & - \\
\hline (r) $\mathrm{Tl}_{03} \mathrm{MoO}_{3}$ & $C 2 / m$ & 183.0 & $(0,0.748,1 / 2)$ & & & \\
\hline${ }^{(s)} \mathrm{Li}_{0.9} \mathrm{Mo}_{6} \mathrm{O}_{17}$ & $P 2_{1} / m$ & 24.0 & & & & \\
\hline \multirow[t]{2}{*}{${ }^{(t)} \eta-\mathrm{Mo}_{4} \mathrm{O}_{11}$} & $P 2_{1} / a$ & 109.0 & $(0,0.2335,0)$ & - & - & \\
\hline & & 35.0 & $(?, 0.42,0.28)$ & _- & _- & \\
\hline${ }^{(t)} \gamma-\mathrm{Mo}_{4} \mathrm{O}_{11}$ & $P n 2_{1} a$ & 100.0 & $(0,0.23,0)$ & _ & _- & \\
\hline${ }^{(u)} \mathrm{Ho}_{5} \mathrm{Ir}_{4} \mathrm{Si}_{10}$ & $\mathrm{P} 4 / \mathrm{mbm}$ & 161.4 & $\left(0,0, \frac{1}{2}\right),\left(0,0, \frac{1}{4} \pm \delta\right)$ & - & 90.0 & $\left(0,0, \frac{1}{2}\right),\left(0,0, \frac{1}{4}\right)$ \\
\hline${ }^{(u)} \mathrm{Er}_{5} \mathrm{Ir}_{4} \mathrm{Si}_{10}$ & $\mathrm{P} 4 / \mathrm{mbm}$ & 151.0 & $\left(0,0, \frac{1}{2}\right),\left(0,0, \frac{1}{4} \pm \delta\right)$ & - & 55.0 & $\left(0,0, \frac{1}{2}\right),\left(0,0, \frac{1}{4}\right)$ \\
\hline (u) $\mathrm{Tm}_{5} \mathrm{Ir}_{4} \mathrm{Si}_{10}$ & $\mathrm{P} 4 / \mathrm{mbm}$ & 134.1 & $\left(0,0, \frac{1}{2}\right),\left(0,0, \frac{1}{4} \pm \delta\right)$ & _- & 111.5 & $\left(0,0, \frac{1}{2}\right),\left(0,0, \frac{1}{)},\left(0,0, \frac{1}{2}\right)\right.$ \\
\hline (u) $\mathrm{Lu}_{5} \mathrm{Ir}_{4} \mathrm{Si}_{10}$ & $\mathrm{P} 4 / \mathrm{mbm}$ & 83.0 & $\left(0,0, \frac{2}{7}\right)$ & - & - & \\
\hline (u) $\left(\mathrm{Lu}_{034} \mathrm{Er}_{066}\right)_{5} \mathrm{Ir}_{4} \mathrm{Si}_{10}$ & $\mathrm{P} 4 / \mathrm{mbm}$ & 102.1 & $\left(0,0, \frac{2}{2}\right)$ & _- & & \\
\hline (u) $\left(\mathrm{Lu}_{0.16} \mathrm{Er}_{0.84}\right)_{5} \mathrm{Ir}_{4} \mathrm{Si}_{10}$ & $\mathrm{P} 4 / \mathrm{mbm}$ & 129.7 & $\left(0,0, \frac{1}{2}\right),\left(0,0, \frac{1}{4} \pm \delta_{1}\right)$ & - & 71.0 & $\left(0,0, \frac{1}{2}\right),\left(0,0, \frac{1}{4}\right),\left(0,0, \frac{1}{8} \pm \delta_{2}\right)$ \\
\hline
\end{tabular}

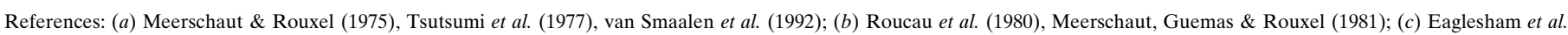

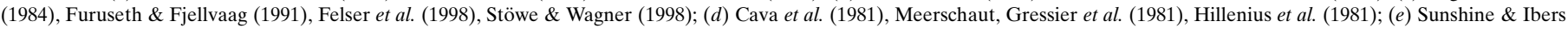

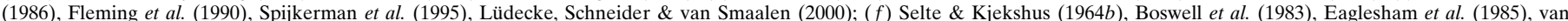

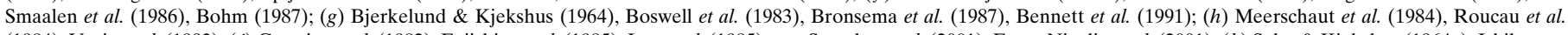

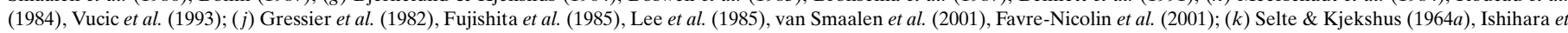

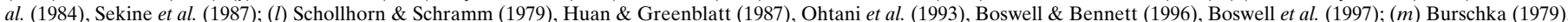

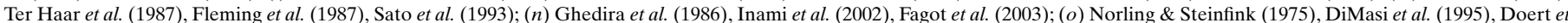

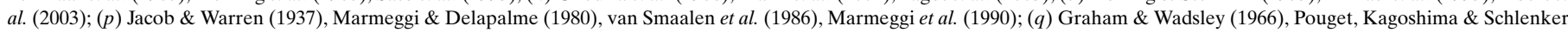

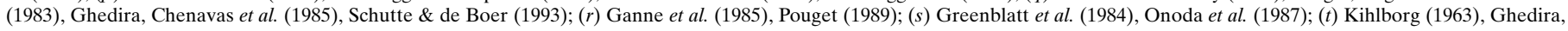
Vincent et al. (1985), Guyot et al. (1985), Negishi et al. (2003); (u) Shelton et al. (1986), Becker et al. (1999), Galli et al. (2000), Kuo et al. (2003), van Smaalen et al. (2004).

\subsection{Modulated atomic structure of the CDW state}

The modulated structure (PLD) in the CDW state can be determined from a sufficient number of integrated intensities of main and satellite Bragg reflections, employing the superspace approach (de Wolff et al., 1981; Janssen et al., 1992; van Smaalen, 1995). The crystal structure is described by three basic structure coordinates $\left(x_{i}^{0}, i=1,2,3\right)$ and a set of Fourier amplitudes $\left(A_{i}^{n}, B_{i}^{n}\right)$ for each independent atom in the average unit cell. As many harmonics $n$ of amplitudes can be refined as there are observed orders $m$ of satellite reflections. The PLD thus is characterized by up to $6 n$ independent parameters for each crystallographically independent atom in the basic structure unit cell. Symmetry relations between parameters of one atom and between different atoms are provided by superspace groups.

The driving force for the formation of the CDW is the gain in electronic energy. The latter increases with the increase of the PLD. If one applies the principle of action and reaction, it is thus likely that the largest modulation amplitudes will occur for atoms carrying the $1 \mathrm{D}$ electron band, while the other atoms assume shifts to minimize the elastic strain energy. Accordingly, the PLD provides information on the location of the CDW in the unit cell, i.e. it points towards the atoms carrying the 1D electron band. Furthermore, it may differentiate between CDWs on pure 1D chains of atoms and those involving a more complicated coupling between PLD and CDW in the conduction band (\$4). 
$\mathrm{NbSe}_{3}$ provides a particularly clear example of coupling between PLD and CDW. A full structure determination by superspace analysis at $T=20 \mathrm{~K}$ of both independent CDWs (Fig. 5) found longitudinal modulation waves on chains of atoms Nb3 for the $\mathbf{q}^{1} \mathrm{CDW}$ and on chains of Nb1 for the $\mathbf{q}^{2}$ CDW (Fig. 9) (van Smaalen et al., 1992). This result was in complete accordance with models for the $\mathrm{CDW}$ in $\mathrm{NbSe}_{3}$ based on theoretical considerations of the basic crystal structure at room temperature and NMR experiments (Wilson, 1979; Ross et al., 1986). However, the modulated structure also indicated contributions of Se atoms to the PLD, including $\mathrm{Se}$ atoms of the inactive $\mathrm{Nb} 2$ chains, which are directly bonded to either Nb1 or Nb3. This observation then provided an explanation for observations in scanning tunneling microscopy (STM) of modulations on the type 2 chains, despite the fact that $\mathrm{Nb} 2$ atoms do not contribute to the CDW (Dai et al., 1991; van Smaalen et al., 1991).

\section{Quasi-one-dimensional electronic crystals}

\subsection{D features in inorganic CDW crystals}

The first discovery of a CDW instability was made on $\mathrm{K}_{2} \mathrm{Pt}(\mathrm{CN})_{4} \mathrm{Br}_{0.30} \cdot 3 \mathrm{H}_{2} \mathrm{O}$ (KCP). Although 1D diffuse scattering at room temperature was found to condense into broad reflections at low temperatures, a phase transition towards a state with long-range order was never observed in this compound (Comes et al., 1973; Renker et al., 1974). The absence of a phase transition can be related to the high degree of one-dimensionality of $\mathrm{KCP}$ and related compounds (Williams et al., 1983). Chains of conducting Pt atoms form the central axis of stacks of flat $\mathrm{Pt}(\mathrm{CN})_{4}$ groups, which are separated by $\mathrm{K}$ and $\mathrm{Br}$ ions and water molecules. The 1D conducting chains of $\mathrm{Pt}$ atoms are thus well separated from each other, and direct electronic interchain interactions will be small, while disorder of the anions and water molecules prevent strong interchain interactions of the lattice type.

Since KCP, many compounds have been synthesized that do exhibit a phase transition from a normal state at high temperatures towards an ordered CDW state at low temperatures. Compounds in the class of 1D organic conductors usually comprise stacks of flat organic molecules that form conducting chains. These stacks may be separated by simple ions or stacks of positive and negative molecular ions may alternate. Conducting 'chains' are now formed by mol-

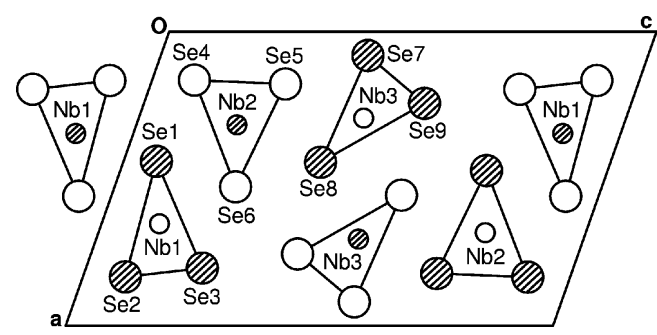

Figure 9

Projections of one unit cell of the basic structure of $\mathrm{NbSe}_{3}$. Hatched and open circles are atoms at $z=0$ and 0.5 , respectively. ecular orbitals extending over all of the flat molecules, and interchain interactions can be much stronger than in KCP. For further details, we refer to the literature (Pouget, 2004, and references therein).

Conducting 'chains' are realized in different ways in inorganic compounds (Table 1). Transition-metal selenides $\left(M \mathrm{Se}_{4}\right)_{n} \mathrm{I}(M=\mathrm{Nb}, \mathrm{Ta} ; n=2,10 / 3)$ and tellurides $M \mathrm{Te}_{4}$ contain chains of metal atoms $M$ parallel to the tetragonal axes (Fig. 10). Refinements of the modulated structures in the CDW states indicated that the PLD is a longitudinal wave on the chains of metal atoms, thus showing that these chains carry the CDW. A similar feature was found for the CDWs in $\mathrm{NbSe}_{3}$ (Fig. 9), and presumably this is also valid for $m-\mathrm{TaS}_{3}, o-\mathrm{TaS}_{3}$ and $\mathrm{ZrTe}_{3}$.

The crystal structure of $\mathrm{Nb}_{3} \mathrm{Te}_{4}$ contains zigzag chains of $\mathrm{Nb}$ atoms as well as open channels parallel to the hexagonal axis. Metal atoms $M=\mathrm{In}, \mathrm{Tl}, \ldots$ can be intercalated into these channels to form $M_{x} \mathrm{Nb}_{3} \mathrm{Te}_{4}$ with $0<x<2$. Intercalation leads to changes of the modulation wavevectors and transition temperatures, but it does not destroy the CDW transition. Presumably, the 1D metallic system is formed by the zigzag chains of $\mathrm{Nb}$ atoms, but structural evidence for this proposition has not yet been obtained, and a contribution of orbitals of Te atoms to the metallic bands cannot be excluded.

The crystal structures of the blue bronzes (e.g. $\left.\mathrm{K}_{0.30} \mathrm{MoO}_{3}\right)$ have slabs of edge-sharing and vertex-sharing $\mathrm{MoO}_{6}$ octahedra that are separated by $\mathrm{K}$ atoms. Columns of about three octahedra wide can be recognized within the layers that would support the $1 \mathrm{D}$ electron bands, but consideration of the basic crystal structure is not sufficient to obtain the exact location of the CDW. The modulated structure of the CDW state has revealed that the $1 \mathrm{D}$ electronic system carrying the $\mathrm{CDW}$ is a combination of Mo- and $\mathrm{O}$-atom orbitals, and that it is not confined to a chain of atoms.

Both forms of $\mathrm{Mo}_{4} \mathrm{O}_{11}$ comprise slabs of vertex-sharing $\mathrm{MoO}_{6}$ octahedra that are separated by $\mathrm{MoO}_{4}$ tetrahedra. The slabs form 2D electronic systems, but the CDWs have been found to be $1 \mathrm{D}$ modulations as opposed to $2 \mathrm{D}$ CDWs in the phosphate bronzes with similar structural features $(\$ 5)$.

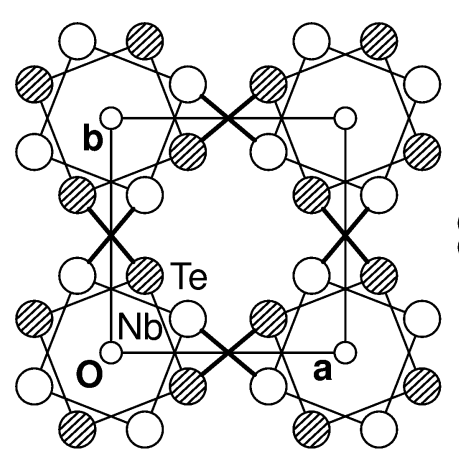

(a)

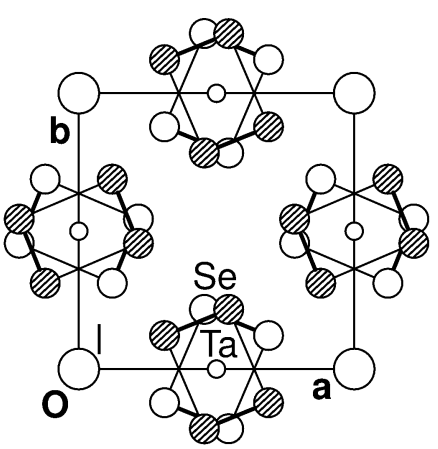

(b)
Figure 10

Projections of the basic structures of $(a) \mathrm{NbTe}_{4}$ and (b) $\left(\mathrm{TaSe}_{4}\right)_{2} \mathrm{I}$. Hatched and open circles are Te/Se atoms at $z=0$ and 0.5 , respectively. $\mathrm{Nb} / \mathrm{Ta}$ are at $z=0.25$ and 0.75 , and $\mathrm{I}$ is at $z= \pm 0.15$. 


\subsection{Location of the CDW}

Although low-dimensional electronic features can be rationalized by consideration of the crystal structures in the normal state, 1D structural features do not imply a CDW phase transition. First of all, this is shown by the many compounds that have been synthesized with crystal structures similar to compounds in Table 1, but that do not have a Peierls transition. These compounds include $\left(\mathrm{NbSe}_{4}\right)_{n} \mathrm{I}(n=3)$, which does not have a CDW transition (Izumi et al., 1984), but differs structurally from the $n=2$ and $n=10 / 3$ compounds only in the average density of iodine atoms in the chains of iodine parallel to $\mathbf{c}$. The class of compounds $M X_{3}(M=\mathrm{Ti}, \mathrm{Zr}, \mathrm{Hf}, \mathrm{Nb}$, $\mathrm{Ta} ; X=\mathrm{S}, \mathrm{Se}, \mathrm{Te}$ ) contains more compounds without a CDW than compounds with a CDW at low temperatures (Meerschaut \& Rouxel, 1986). They have related structures, whereby the principal building block is given by a pair of trigonal prismatic $M X_{3}$ chains that carry the CDWs in $\mathrm{NbSe}_{3}$ (Fig. 9). Secondly, many ternary and quaternary compounds have been synthesized with low-dimensional structures, but without CDWs (e.g. see Mitchell \& Ibers, 2002).

Obviously, more stringent criteria are needed to predict whether a crystal will undergo a Peierls transition at low temperatures. One such criterion is that the compound needs to have metallic conductivity for the Peierls mechanism to work. Indeed, many low-dimensional compounds without a CDW state are semiconductors in their normal state. Although isostructural to CDW compounds, the different electronic properties can be explained by different electronic structures of the elements when they replace each other in the same structure type. However, 1D compounds with metallic conductivity exist that do not develop a CDW, e.g. $\mathrm{TaSe}_{3}$ (Meerschaut \& Rouxel, 1986), and metallic conductivity or a particularly high anisotropy of the conductivity are not good criteria to distinguish compounds with a CDW from those without a CDW transition.

Electronic band-structure calculations for the basic crystal structures provide information about the metallic character, possible nesting conditions of the Fermi surface and the contributions of different atoms to states at the Fermi level. They are perfectly suitable for a rationalization of the properties of CDW compounds, but they are not sufficiently accurate to predict the Peierls transition. This can be understood from the limited accuracy of band-structure calculations, e.g. correlation effects are not taken into account. Furthermore, they do not provide information about the electronphonon coupling, and thus they might fail to predict a possible Peierls transition in principle.

Similarities between basic structures are not sufficient to conclude that they have similar CDW properties, even if a $\mathrm{CDW}$ is known to develop in both compounds. An example is provided by the compounds $\mathrm{NbSe}_{3}, \mathrm{FeNb}_{3} \mathrm{Se}_{10}$ and $\mathrm{NiTa}_{2} \mathrm{Se}_{7}$. $\mathrm{NbSe}_{3}$ develops two independent CDWs (Fig. 5) on the pairs of trigonal prismatic coordinated chains of $\mathrm{Nb} 3$ and $\mathrm{Nb} 1$, respectively (Fig. 9). Based on consideration of the electronic band structure of the basic crystal structure, Wilson (1979) had predicted the locations of both CDWs. His argument involved the shortest $\mathrm{Se}-\mathrm{Se}$ distances of each trigonal prismatic column. The distance between $\mathrm{Se} 7$ and $\mathrm{Se} 9$ is $2.37 \AA$, indicating an $\mathrm{Se}_{2}^{2-}$ complex ion. Consequently, $\mathrm{Nb} 3$ has a partially filled $d$ band and is a 1D metal. On the other hand, the distance between Se 4 and Se 5 is $2.92 \AA$, which corresponds to the presence of two $\mathrm{Se}^{2-}$ ions. Accordingly, $\mathrm{Nb} 2$ is fully ionized and insulating, and thus cannot participate in the formation of a CDW. The distance between $\mathrm{Se} 2$ and $\mathrm{Se} 3$ is $2.48 \AA$, and the pair $\mathrm{Se} 2-\mathrm{Se} 3$ is intermediate between $\mathrm{Se}_{2}^{2-}$ and $\mathrm{Se}^{2-}$. Wilson (1979) concluded that $\mathrm{Nb3}$ supports the $\mathbf{q}^{1}$-type CDW (highest $T_{\mathrm{CDW}}$ and largest gain in electronic energy), and that $\mathrm{Nb} 1$ supports the $\mathbf{q}^{2}$-type $\mathrm{CDW}$, while $\mathrm{Nb} 2$ does not participate in the CDWs, in accordance with experiments.

Both $\mathrm{FeNb}_{3} \mathrm{Se}_{10}$ and $\mathrm{NiTa}_{2} \mathrm{Se}_{7}$ contain pairs of trigonal prismatic chains next to metal atoms in distorted octahedral coordination (Fig. 11). The distance between $\mathrm{Se} 2$ and $\mathrm{Se} 3$ in $\mathrm{FeNb}_{3} \mathrm{Se}_{10}$ is $2.34 \AA$, showing that the trigonal prismatic columns are similar to the Nb3-type columns in $\mathrm{NbSe}_{3}$. A Peierls transition with $T_{\mathrm{CDW}}=140 \mathrm{~K}$ has been found in $\mathrm{FeNb}_{3} \mathrm{Se}_{10}$, close to $T_{\mathrm{CDW} 1}$ of $\mathrm{NbSe}_{3}$, and it was suggested that the CDW must be located on the trigonal prismatic columns (Rouxel, 1986), although experimental evidence is still lacking.

The distance between $\mathrm{Se} 2$ and $\mathrm{Se} 5$ in $\mathrm{NiTa}_{2} \mathrm{Se}_{7}$ is $2.54 \AA$, similar to the $\mathrm{Se} 2-\mathrm{Se} 3$ distance in $\mathrm{NbSe}_{3}$. Together with $T_{\mathrm{CDW}}=54 \mathrm{~K}$ in $\mathrm{NiTa}_{2} \mathrm{Se}_{7}$, this suggests a similarity between the CDW in $\mathrm{NiTa}_{2} \mathrm{Se}_{7}$ and the $\mathbf{q}^{2}$-type CDW of $\mathrm{NbSe}_{3}$ on the Nb1-type columns. However, the modulated structure of the CDW state of $\mathrm{NiTa}_{2} \mathrm{Se}_{7}$ shows that the primary CDW (first harmonic modulation wave) is a transverse wave on the $\mathrm{Ni}$ and $\mathrm{Se} 2$ atoms, while the secondary CDW (second harmonic modulations) is located on Ta2. The contribution of trigonal prismatic Ta1 is insignificant, thus providing evidence for an entirely different realization of the CDWs in $\mathrm{NbSe}_{3}$ and $\mathrm{NiTa}_{2} \mathrm{Se}_{7}$.

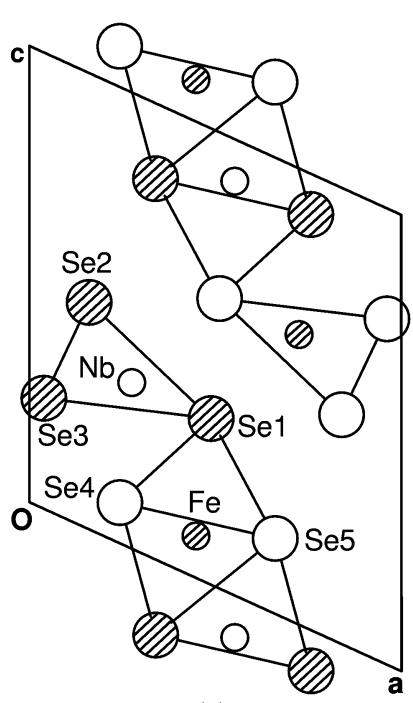

(a)

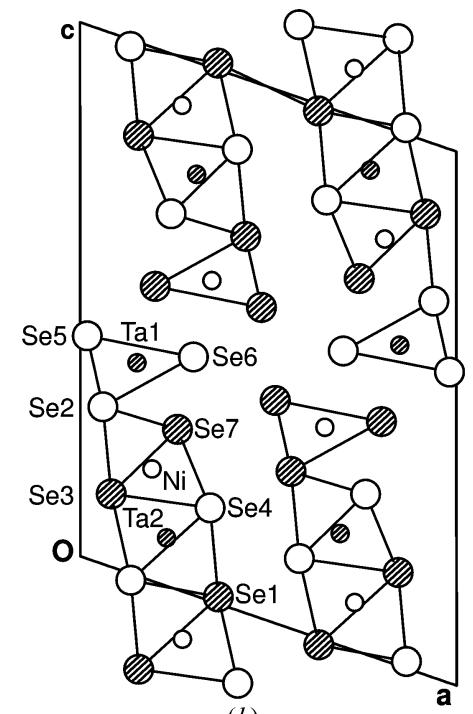

(b)
Figure 11

Projections of one unit cell of the basic structures of $(a) \mathrm{FeNb}_{3} \mathrm{Se}_{10}$ and (b) $\mathrm{NiTa}_{2} \mathrm{Se}_{7}$. 
Table 2

Phase diagrams of inorganic compounds with a 2D CDW.

$T_{c}$ indicates $T_{\mathrm{CDW}}$ or $T_{\text {lock-in }}$; the fourth column gives the space group of the normal phase or supercell or the superspace group of the modulated phase.

\begin{tabular}{|c|c|c|c|}
\hline Compound & $T_{c}(\mathrm{~K})$ & $\mathbf{q}$ & Space group \\
\hline \multirow{4}{*}{ (a) $1 T-\mathrm{TaS}_{2}$} & - & - & $P \overline{3} m 1$ \\
\hline & 543.0 & $(0.283,0,1 / 3)$ & \\
\hline & 350.0 & $(0.245,0.068,1 / 3)$ & $P \overline{3}(\alpha \beta 1 / 3)$ \\
\hline & 180.0 & $(3 / 13,1 / 13,1 / 3)$ & \\
\hline \multirow[t]{3}{*}{ (b) $1 T-\mathrm{TaSe}_{2}$} & - & - & $P \overline{3} m 1$ \\
\hline & $\sim 600$ & $(0.283,0,1 / 3)$ & \\
\hline & 473.0 & $(3 / 13,1 / 13, *)$ & $P \overline{1}$ \\
\hline \multirow[t]{3}{*}{${ }^{(c)} 1 T-\mathrm{VSe}_{2}$} & - & - & $P \overline{3} m 1$ \\
\hline & 110.0 & $(0.25,0,0.314)$ & \\
\hline & 85.0 & $(0.25,0,0.309)$ & \\
\hline \multirow{2}{*}{ (d) $2 \mathrm{H}-\mathrm{TaS}_{2}$} & - & - & $P 6_{3} / m m c$ \\
\hline & 75.0 & $(0.339,0,0)$ & \\
\hline \multirow[t]{2}{*}{ (e) $2 \mathrm{H}-\mathrm{NbSe}_{2}$} & - & - & $P 6_{3} / m m c$ \\
\hline & 33.5 & $(0.327,0,0)$ & \\
\hline \multirow[t]{3}{*}{ (e) $2 \mathrm{H}-\mathrm{TaSe}_{2}$} & - & - & $P 6_{3} / m m c$ \\
\hline & 122.3 & $(0.327,0,0)$ & \\
\hline & 90.0 & $(1 / 3,0,0)$ & \\
\hline \multirow[t]{3}{*}{ (f) $4 H_{b}-\mathrm{TaS}_{2}$} & - & - & $P 6_{3} / m m c$ \\
\hline & - & $(\sim 0.265,0,0)$ & \\
\hline & 315.0 & $(3 / 13,1 / 13,0)$ & \\
\hline \multirow{4}{*}{ (f) $4 H_{b}-\mathrm{TaSe}_{2}$} & - & - & $P 6_{3} / m m c$ \\
\hline & 600.0 & $(0.265,0,0)$ & \\
\hline & 410.0 & $(3 / 13,1 / 13,0)$ & $P 6_{3} / m(\alpha \beta 0)$ \\
\hline & 75.0 & $(0.347,0,0)$ & \\
\hline \multirow[t]{3}{*}{${ }^{(g),(h)}\left(\mathrm{PO}_{2}\right)_{4}\left(\mathrm{WO}_{3}\right)_{8}$} & - & - & $P 2_{1} 2_{1} 2_{1}$ \\
\hline & 80.0 & $(0.330,0.295,0)$ & \\
\hline & 52.0 & $(0.340,0,0)$ & $P 2_{1} 2_{1} 2_{1}(\alpha \beta 0)$ \\
\hline \multirow{3}{*}{${ }^{(g),(j)}\left(\mathrm{PO}_{2}\right)_{4}\left(\mathrm{WO}_{3}\right)_{12}$} & - & - & $P 2_{1} 2_{1} 2_{1}$ \\
\hline & 120.0 & $(0.385,0, ?)$ & \\
\hline & 62.0 & $(0.310,0.295, ?)$ & \\
\hline \multirow{2}{*}{${ }^{(g),(k)}\left(\mathrm{PO}_{2}\right)_{4}\left(\mathrm{WO}_{3}\right)_{20}$} & - & - & Pmmm \\
\hline & 450.0 & $(3 / 7,0,0)$ & $P 2_{1}(\alpha 00)$ \\
\hline
\end{tabular}

References: (a) Jellinek (1962), Scruby et al. (1975), Brouwer (1978), Yamamoto et al. (1990), Spijkerman et al. (1997); (b) Kadijk et al. (1964), Wilson et al. (1975), Brouwer \& Jellinek (1980), Wiegers et al. (2001); (c) Tsutsumi (1982); (d) Scholz et al. (1982); (e) Moncton et al. (1977), Fleming et al. (1984), Brouwer \& Jellinek (1980); $(f)$ Wilson et al. (1975), Salvo et al. (1976), Ludecke et al. (1999); $(g)$ an extensive review of basic structures and $\mathrm{CDW}$ transitions of the phosphate bronzes $\left(\mathrm{PO}_{2}\right)_{4}\left(\mathrm{WO}_{3}\right)_{2 m}(2<m<14)$ is given by Roussel, Labbé \& Groult (2000); here a selection of compounds is given; $(h)$ Giroult et al. (1981), Foury \& Pouget (1993), Lüdecke, Jobst \& van Smaalen (2000), Ludecke et al. (2001); ( $j$ ) Labbe et al. (1986), Foury \& Pouget (1993); ( $k$ ) Ottolenghi \& Pouget (1996), Roussel, Labbé, Leligny et al. (2000).

\section{Competition between 1D and 2D CDWs}

Two-dimensional CDWs have been found in several classes of compounds (Table 2). Most extensively studied are the transition-metal dichalcogenides $M X_{2}(M=\mathrm{Nb}, \mathrm{Ta} ; X=\mathrm{S}, \mathrm{Se})$, which are layered compounds known for their polytypism (Jellinek, 1962). $M$ atoms are at the nodes of a planar hexagonal lattice, and they are sandwiched between planes of $X$ atoms, with either trigonal prismatic coordination of $M(T$ layers) or octahedral coordination ( $H$ layers). Different sequences of $H$ and $T$ layers result in structures for which one translational period encompasses between 1 and 6 layers of $M X_{2}$, thus defining the different polytypes (Fig. 12). Most polytypes are stable in some interval at high temperatures, and the CDW phenomena pertain to phase transitions within metastable states for most of the $M X_{2}$ compounds.

Like 1D metals $(\S 4)$, CDW transitions in 2D electronic compounds can be explained by nesting of the Fermi surfaces
(Wilson et al., 1975; Friend \& Yoffe, 1987). The planes of metal atoms support $2 \mathrm{D}$ electron bands. The latter are dispersionless in the direction perpendicular to the layers, resulting in Fermi surfaces that are topologically equivalent to cylinders. However, their true shapes have flat portions along in-layer directions too, resulting in nesting vectors $\mathbf{q}^{1}=(\alpha 00)$ or $\mathbf{q}^{1^{\prime}}=(\alpha \beta 0)$ (Fig. 13). A possible non-zero component along $\mathbf{c}^{*}$ pertains to different stackings of the 2D CDWs (Table 2).

The threefold rotational symmetry of the basic structures defines for each nesting vector three symmetry-equivalent modulation wavevectors that provide equally good nesting conditions. Only two of these vectors are independent (e.g. $\mathbf{q}^{1}=(\alpha 00), \mathbf{q}^{2}=(0 \alpha 0)$ and $\left.\mathbf{q}^{3}=\mathbf{q}^{2}-\mathbf{q}^{1}\right)$. For $\mathbf{q}^{1^{\prime}}=(\alpha \beta 0)$, the mirror planes define an additional three symmetry-equivalent vectors, resulting in a total of six equivalent vectors, of which three are independent. Thus, $2 \mathrm{D}$ or 3D CDWs are formed if all nesting vectors are simultaneously excited. However, it is known from the Landau theory of phase transitions (Toledano \& Toledano, 1987) that the low-symmetry phase (CDW state) may be modulated with less than the complete number of symmetry-equivalent wavevectors, depending on the relative importance of the different interactions in the crystal.

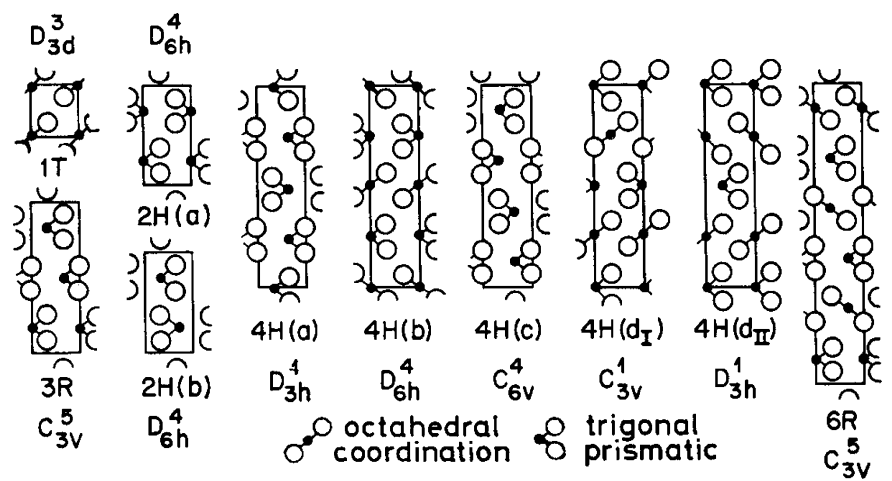

Figure 12

Sections (110) of the hexagonal unit cells of various polytypes of layered transition-metal dichalcogenides $M X_{2}$. Reprinted with permission from Wilson et al. (1975). Adv. Phys. 24, 117-201. Copyright (1975) Taylor \& Francis Ltd, http://www.tandf.co.uk/journals.

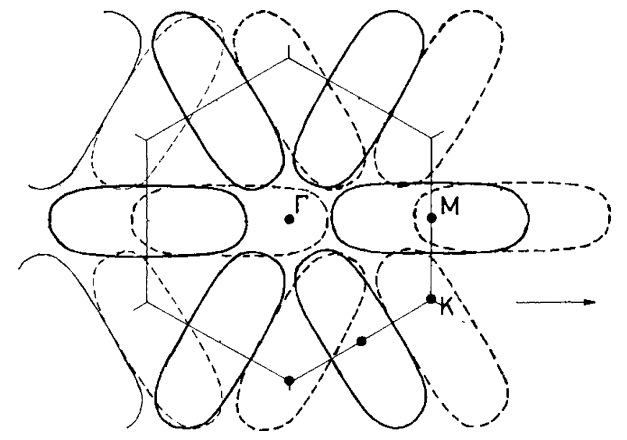

Figure 13

Section of the Fermi surface of $1 T-\mathrm{TaSe}_{2}$ parallel to the hexagonal plane. Coincidence of the Fermi surface (full lines) and one displaced along $\Gamma M$ (dashed lines) demonstrate the nesting condition for $\mathbf{q}=(\alpha 00)$. Reprinted with permission from Wilson et al. (1975). Adv. Phys. 24, 117-201. Copyright (1975) Taylor \& Francis Ltd, http://www.tandf.co.uk/ journals. 
Accordingly, 1D or 2D CDWs are formed and the point symmetry is lowered at the CDW transitions, as has been observed for several compounds (Table 2). As a consequence, most compounds are twinned in their CDW state, with twinning operators provided by the lost rotational symmetry elements.

CDWs in $M X_{2}$ compounds have been studied by various diffraction techniques and they have been directly imaged by STM (Coleman et al., 1988; Thomson et al., 1994). More recently, the dispersion relations of electron bands near the Fermi surface have been measured by angle-resolved photoelectron spectroscopy (ARPES). These experiments have been used to study the mechanism of CDW formation. Both Fermi surface nesting (Straub et al., 1999) and alternative mechanisms have been proposed (Rice \& Scott, 1975; Pillo et al., 2000; Liu et al., 2000).

Structure refinements against X-ray diffraction data of several compounds have shown that the characteristic building block of the CDW states is a $2 \mathrm{D}$ cluster of $13 \mathrm{M}$ atoms with pseudo-sixfold symmetry (Brouwer \& Jellinek, 1980). Different modulations pertain to a different $3 \mathrm{D}$ ordering of these clusters (Table 2). Notably, 2D CDWs are formed in $1 T-\mathrm{TaS}_{2}$ and $4 H_{b}-\mathrm{TaSe}_{2}$, while a $1 \mathrm{D} \mathrm{CDW}$ is formed in the commensurate state of $1 T-\mathrm{TaSe}_{2}$, resulting in multiply twinned crystals.

Competition between multidimensional CDWs and 1D CDWs can also be recognized amongst the phosphate bronzes, where both 1D and 2D CDWs have been found (Table 2), while similar 2D metallic layers in $\mathrm{Mo}_{4} \mathrm{O}_{11}$ give rise to $1 \mathrm{D}$ CDWs only (Table 1 ).

\section{Conclusions}

One-dimensional electrical conductors behave qualitatively different from 3D metals. Particularly interesting are compounds with incommensurate charge-density waves (CDWs), because the latter are responsible for non-linear electrical conductivity. Many 1D electronic crystals have been synthesized that undergo a phase transition towards a lowtemperature CDW state.

X-ray diffraction has proven to be a universal tool for studying the properties of CDWs. Both fluctuations in the normal state, the evolution of the CDW amplitude with temperature and strain of the $\mathrm{CDW}$ in the non-linear conducting regime, have been measured quantitatively by $\mathrm{X}$-ray diffraction. Crystal structure determinations of the periodic structures of the normal states, and of the incommensurately modulated structures of the CDW states, have provided important information for the understanding of physical properties of CDWs, if only as the basis for electronic band-structure calculations. A long-standing problem is the computation of band structures of incommensurate crystals that would allow direct information to be obtained on the nature of the gap in the electronic structure of the CDW state.

Theories describing CDW properties have often been tested against data on a very few compounds only, and they do not necessarily apply in the general case. Experimental studies can help here by providing detailed information on a variety of compounds. An open question is the understanding of the physical properties of $1 \mathrm{D}$ electronic systems and CDW transitions in compounds with strongly correlated electrons or large interchain interactions (Becker et al., 1999).

Financial support for this research was obtained from the Deutsche Forschungsgemeinschaft (DFG).

\section{References}

Becker, B., Patil, N. G., Ramakrishnan, S., Menovsky, A. A., Nieuwenhuys, G. J., Mydosh, J. A., Kohgi, M. \& Iwasa, K. (1999). Phys. Rev. B, 59, 7266-7269.

Bennett, J. C., Boswell, F. W., Prodan, A., Corbett, J. M. \& Ritchie, S. (1991). J. Phys. Condens. Matter, 3, 6959-6972.

Bjerkelund, E. \& Kjekshus, A. (1964). Z. Anorg. Allg. Chem. 328, 235-242.

Bohm, H. (1987). Z. Kristallogr. 180, 113-122.

Boswell, F. W. \& Bennett, J. C. (1996). Mater. Res. Bull. 31, 1083-1092.

Boswell, F. W., Prodan, A. \& Brandon, J. K. (1983). J. Phys. C, 16, 1067-1076.

Boswell, F. W., Scholz, G. A. \& Bennett, J. C. (1997). Phys. Rev. B, 56, 1175-1178.

Brazovskii, S., Kirova, N. \& Monceau, P. (2002). Editors. Proc. International Workshop on Electronic Crystals. J. Phys. (Paris) IV, Vol. 12, Pr 9.

Bronsema, K. D., van Smaalen, S., de Boer, J. L., Wiegers, G. A., Jellinek, F. \& Mahy, J. (1987). Acta Cryst. B43, 305-313.

Brouwer, R. (1978). PhD thesis, University of Groningen, The Netherlands.

Brouwer, R. \& Jellinek, F. (1980). Physica (Utrecht), B99, 51-55.

Burschka, C. (1979). Z. Naturforsch. Teil B, 34, 675-677.

Canadell, E., Rachidi, E. I., Pouget, J. P., Gressier, P., Meerschaut, A., Rouxel, J., Jung, D., Evain, M. \& Whangbo, M. H. (1990). Inorg. Chem. 29, 1401-1407.

Cava, R. J., Himes, V. L., Mighell, A. D. \& Roth, R. S. (1981). Phys. Rev. B, 24, 3634-3637.

Chaussy, J., Haen, P., Lasjaunias, J. C., Monceau, P., Waysand, G., Waintal, A., Meerschaut, A., Molinié, P. \& Rouxel, J. (1976). Solid State Commun. 20, 759-763.

Claessen, R., Sing, M., Schwingenschlogl, U., Blaha, P., Dressel, M. \& Jacobsen, C. S. (2002). Phys. Rev. Lett. 88, 096402.

Coleman, R. V., Giambattista, B., Hansma, P. K., Johnson, A., McNairy, W. W. Slough, C. G. (1988). Adv. Phys. 37, 559-644.

Comes, R., Lambert, M., Launois, H. \& Zeller, H. R. (1973). Phys. Rev. B, 8, 571-575.

Dai, Z., Slough, C. G. \& Coleman, R. V. (1991). Phys. Rev. Lett. 66, 1318-1321.

DiCarlo, D., Sweetland, E., Sutton, M., Brock, J. D. \& Thorne, R. E. (1993). Phys. Rev. Lett. 70, 845-848.

DiMasi, E., Aronson, M. C., Mansfield, J. F., Foran, B. \& Lee, S. (1995). Phys. Rev. B, 52, 14516-14525.

Doert, T., Polequin Fokwa Tsinde, B., Simon, P., Lidin, S. \& Sohnel, T. (2003). Chem. Eur. J. 9, 5865-5872.

Eaglesham, D. J., Bird, D., Withers, R. L. \& Steeds, J. W. (1985). J. Phys. C, 18, 1-11.

Eaglesham, D. J., Steeds, J. W. Wilson, J. A. (1984). J. Phys. C, 17, L697-L698.

Fagot, S., Foury-Leylekian, P., Ravy, S., Pouget, J. P. \& Berger, H. (2003). Phys. Rev. Lett. 90, 196401.

Favre-Nicolin, V., Bos, S., Lorenzo, J. E., Hodeau, J.-L., Berar, J.-F., Monceau, P., Currat, R., Levy, F. \& Berger, H. (2001). Phys. Rev. Lett. 87, 015502.

Felser, C., Finckh, E. W., Kleinke, H., Rocker, F. \& Tremel, W. (1998). J. Mater. Chem. 8, 1787-1798. 
Fleming, R. M. (1980). Phys. Rev. B, 22, 5606-5612.

Fleming, R. M. \& Grimes, C. C. (1979). Phys. Rev. Lett. 42, 1423-1426.

Fleming, R. M., Moncton, D. E., Axe, J. D. \& Brown, G. S. (1984). Phys. Rev. B, 30, 1877-1883.

Fleming, R. M., Sunshine, S. A., Chen, C. H., Schneemeyer, L. F. \& Waszczak, J. V. (1990). Phys. Rev. B, 42, 4954-4959.

Fleming, R. M., Ter Haar, L. W. \& DiSalvo, F. J. (1987). Phys. Rev. B, 35, 5388-5391.

Foury, P. \& Pouget, J. P. (1993). Int. J. Mod. Phys. B, 7, 3973-4003.

Friend, R. H. \& Yoffe, A. D. (1987). Adv. Phys. 36, 1-94.

Fujishita, H., Sato, M., Sato, S. \& Hoshino, S. (1985). J. Phys. C, 18, $1105-1114$

Furuseth, S. \& Fjellvaag, H. (1991). Acta Chem. Scand. 45, 694-697.

Galli, F., Feyerherm, R., Hendrikx, R. W. A., Dudzik, E., Nieuwenhuys, G. J., Ramakrishnan, S., Brown, S. D., van Smaalen, S. \& Mydosh, J. A. (2002). J. Phys. Condens. Matter, 14, 5067-5075.

Galli, F., Ramakrishnan, S., Taniguchi, T., Nieuwenhuys, G. J., Mydosh, J. A., Geupel, S., Ludecke, J. \& van Smaalen, S. (2000). Phys. Rev. Lett. 85, 158-161.

Ganne, M., Boumaza, A., Dion, M. \& Dumas, J. (1985). Mater. Res. Bull. 20, 1297-1308.

Ghedira, M., Anne, M., Chenavas, J., Marezio, M. \& Sayetat, F. (1986). J. Phys. C, 19, 6489-6503.

Ghedira, M., Chenavas, J., Marezio, M. \& Marcus, J. (1985). J. Solid State Chem. 57, 300-313.

Ghedira, M., Vincent, H., Marezio, M., Marcus, J. \& Furcaudot, G. (1985). J. Solid State Chem. 56, 66-73.

Giroult, J. P., Goreaud, M., Labbé, P. \& Raveau, B. (1981). Acta Cryst. B37, 2139-2142.

Graham, J. \& Wadsley, A. D. (1966). Acta Cryst. 20, 93-100.

Greenblatt, M., McCarroll, W. H., Neifeld, R., Croft, M. \& Waszczak, J. V. (1984). Solid State Commun. 51, 671-674.

Gressier, P., Guemas, L. \& Meerschaut, A. (1982). Acta Cryst. B38, 2877-2879.

Grüner, G. (1994). Density Waves in Solids. Reading, MA: AddisonWesley.

Guyot, H., Schlenker, C., Pouget, J. P., Ayroles, R. \& Roucau, C. (1985). J. Phys. C, 18, 4427-4434.

Hillenius, S. J., Coleman, R. V., Fleming, R. M. \& Cava, R. J. (1981). Phys. Rev. B, 23, 1567-1575.

Huan, G. \& Greenblatt, M. (1987). Mater. Res. Bull. 22, 943-949.

Inami, T., Ohwada, K., Kimura, H., Watanabe, M., Noda, Y., Nakamura, H., Yamasaki, T., Shiga, M., Ikeda, N. Murakami, Y. (2002). Phys. Rev. B, 66, 073108.

Ishihara, Y., Nakada, I., Suzuki, K. \& Ichihara, M. (1984). Solid State Commun. 50, 657-659.

Izumi, M., Iwazumi, T., Kunimitsu, U., Yoshizaki, R. \& Matsuura, E. (1984). Solid State Commun. 51, 191-184.

Jacob, C. W. \& Warren, B. E. (1937). J. Am. Chem. Soc. 59, 2588-2591.

Janssen, T., Janner, A., Looijenga-Vos, A. \& de Wolff, P. M. (1992). In International Tables for Crystallography, Vol. C, edited by A. J. C. Wilson, pp. 797-835. Dordrecht: Kluwer Academic Publishers.

Jellinek, F. (1962). J. Less Common Met. 4, 9-15.

Kadijk, F., Huisman, R. \& Jellinek, F. (1964). Rec. Trav. Chim. PaysBas, 83, 768-775.

Kihlborg, L. (1963). Ark. Kemi, 21, 365-377.

Kuo, Y. K., Hsu, F. H., Li, H. H., Huang, H. L., Huang, C. W., Lue, C. S. \& Yang, H. D. (2003). Phys. Rev. B, 67, 195101.

Labbe, P., Goreaud, M. \& Raveau, B. (1986). J. Solid State Chem. 61, 324-331.

Lee, K. B., Davidov, D. \& Heeger, A. J. (1985). Solid State Commun. 54, 673-677.

Liu, R., Tonjes, W. C., Greanya, V. A., Olson, C. G. \& Frindt, R. F. (2000). Phys. Rev. B, 61, 5212-5216.

Ludecke, J., Jobst, A., Geupel, S. \& van Smaalen, S. (2001). Phys. Rev. $B, \mathbf{6 4}, 104105$.

Lüdecke, J., Jobst, A. \& van Smaalen, S. (2000). Europhys. Lett. 49, 357-361.
Lüdecke, J., Schneider, M. \& van Smaalen, S. (2000). J. Solid State Chem. 153, 152-157.

Ludecke, J., van Smaalen, S., Spijkerman, A., de Boer, J. L. \& Wiegers, G. A. (1999). Phys. Rev. B, 59, 6063-6071.

Magonov, N. \& Whangbo, M.-H. (1996). Surface Analysis with STM and $A F M$. Weinheim: VCH Publishers.

Marmeggi, J. C. \& Delapalme, A. (1980). Physica (Utrecht) B, 102, 309-312.

Marmeggi, J. C., Lander, G. H., van Smaalen, S., Bruckel, T. \& Zeyen, C. M. E. (1990). Phys. Rev. B, 42, 9365-9376.

Meerschaut, A., Gressier, P., Guemas, L. \& Rouxel, J. (1981). Mater. Res. Bull. 16, 1035-1040.

Meerschaut, A., Gressier, P., Guemas, L. \& Rouxel, J. (1984). J. Solid State Chem. 51, 307-314.

Meerschaut, A., Guemas, L. \& Rouxel, J. (1981). J. Solid State Chem. 36, 118-123.

Meerschaut, A. \& Rouxel, J. (1975). J. Less Common Met. 39, 197-203.

Meerschaut, A. \& Rouxel, J. (1986). In Crystal Chemistry and Properties of Materials with Quasi-one-dimensional Structures, edited by J. Rouxel, pp. 205-279. Dordrecht: Reidel.

Mitchell, K. \& Ibers, J. A. (2002). Chem. Rev. 102, 1929-1952.

Monceau, P. (1985). In Electronic Properties of Inorganic Quasi-onedimensional Compounds. Part II. Experimental, edited by $\mathrm{P}$. Monceau, pp. 139-268. Dordrecht: Reidel.

Moncton, D. E., Axe, J. D. \& DiSalvo, F. J. (1977). Phys. Rev. B, 16, 801-819.

Moudden, A. H., Vasiliu-Doloc, L., Monceau, P. \& Levy, F. (1993). J. Phys. (Paris) IV, 3, C2, 121-124.

Negishi, H., Kuroiwa, Y., Akamine, H., Aoyagi, S., Sawada, A., Shobu, T., Negishi, S. \& Sasaki, M. (2003). Solid State Commun. 125, 45-49.

Norling, B. K. \& Steinfink, H. (1975). Inorg. Chem. 5, 1488.

Ohtani, T., Sano, Y. \& Yokota, Y. (1993). J. Solid State Chem. 103, 504-513.

Onoda, M., Toriumi, K., Matsuda, Y. \& Sato, M. (1987). J. Solid State Chem. 66, 163-170.

Ottolenghi, A. \& Pouget, J. P. (1996). J. Phys. (Paris) I, 6, 1059-1083.

Overhauser, A. W. (1971). Phys. Rev. B, 3, 3173-3182.

Peierls, R. E. (1955). Quantum Theory of Solids. Oxford: Clarendon Press.

Pillo, T., Hayoz, J., Berger, H., Fasel, R., Schlapbach, L. \& Aebi, P. (2000). Phys. Rev. B, 62, 4277-4287.

Pouget, J. P. (1989). In Low-dimensional Electronic Properties of Molybdenum Bronzes and Oxides, edited by C. Schlenker, pp. 87-157. Dordrecht: Kluwer Academic Publishers.

Pouget, J. P. (1996). In Physics and Chemistry of Low-dimensional Inorganic Conductors, edited by C. Schlenker, J. Dumas, M. Greenblatt \& S. van Smaalen, pp. 185-217. New York: Plenum Press.

Pouget, J.-P. (2004). Z. Kristallogr. 219, 711-718.

Pouget, J. P., Kagoshima, S. \& Schlenker, C. J., M. (1983). J. Phys. Lett. (Paris), 44, L113-L120.

Pouget, J. P., Khanna, S. K., Denoyer, F., Comes, R., Garito, A. F. \& Heeger, A. J. (1976). Phys. Rev. Lett. 37, 437-440.

Pouget, J. P., Moret, R., Meerschaut, A., Guemas, L. \& Rouxel, J. (1983). J. Phys. (Paris), 44, C3, 1729-1731.

Pouget, J. P., Noguera, C., Moudden, A. H. \& Moret, R. (1985). J. Phys. (Paris), 46, 1731-1742.

Renker, B., Pintschovius, L., Glaser, W., Rietschel, H., Comes, R., Liebert, L. \& Drexel, W. (1974). Phys. Rev. Lett. 32, 836-839.

Requardt, H. F., Nad, Ya., Monceau, P., Currat, R., Lorenzo, J. E., Brazovskii, S., Kirova, N., Grübel, G. \& Vettier, Ch. (1998). Phys. Rev. Lett. 80, 5631-5634.

Rice, T. M. \& Scott, G. K. (1975). Phys. Rev. Lett. 35, 120-123.

Ross, J. H. Jr, Wang, Z. \& Slichter, C. P. (1986). Phys. Rev. Lett. 56, 663-666. 
Roucau, C., Ayroles, R., Gressier, P. \& Meerschaut, A. (1984). J. Phys. C, 17, 2993-2998.

Roucau, C., Ayroles, R., Monceau, P., Guemas, L., Meerschaut, A. \& Rouxel, J. (1980). Phys. Status Solidi A, 62, 483-493.

Roussel, P., Labbé, P. \& Groult, D. (2000). Acta Cryst. B56, 377-391.

Roussel, P., Labbé, P., Leligny, H., Groult, D., Foury-Leylekian, P. \& Pouget, J. P. (2000). Phys. Rev. B, 62, 176-188.

Rouxel, J. (1986). J. Solid State Chem. 64, 305-321.

Salvo, F. J. D., Moncton, D. E., Wilson, J. A. \& Mahajan, S. (1976). Phys. Rev. B, 14, 1543-1546.

Sato, H., Kojima, N. \& Kagoshima, S. (1993). J. Phys. Soc. Jpn, 62, 2051-2061.

Schafer, J., Rotenberg, E., Kevan, S. D., Blaha, P., Claessen, R. \& Thorne, R. E. (2001). Phys. Rev. Lett. 87, 196403.

Schollhorn, R. \& Schramm, W. (1979). Z. Naturforsch. Teil B, 34, 697-699.

Scholz, G. A., Singh, O., Frindt, R. F. \& Curzon, A. E. (1982). Solid State Commun. 44, 1455-1459.

Schutte, W. J. \& de Boer, J. L. (1993). Acta Cryst. B49, 579-591.

Scruby, C. B., Williams, P. M. \& Parry, G. S. (1975). Philos. Mag. 31, 225.

Sekine, T., Yoshinari, K., Matsuura, E., Uchinokura, K. \& Yoshizaki, R. (1987). Phys. Rev. B, 36, 3153-3160.

Selte, K. \& Kjekshus, A. (1964a). Acta Cryst. 17, 1568-1572.

Selte, K. \& Kjekshus, A. (1964b). Acta Chem. Scand. 18, 692-696.

Shelton, R. N., Hausermann-Berg, L. S., Klavins, P., Yang, H. D., Anderson, M. S. Swenson, C. A. (1986). Phys. Rev. B, 34, 4590-4594.

Smaalen, S. van (1995). Crystallogr. Rev. 4, 79-202.

Smaalen, S. van, de Boer, J. L., Coppens, P. \& Graafsma, H. (1991). Phys. Rev. Lett. 67, 1471.

Smaalen, S. van, Bronsema, K. D. \& Mahy, J. (1986). Acta Cryst. B42, 43-50.

Smaalen, S. van, de Boer, J. L., Meetsma, A., Graafsma, H., Sheu, H.-S., Darovskikh, A., Coppens, P. \& Levy, F. (1992). Phys. Rev. B, 45, 3103-3106.

Smaalen, S. van, Lam, E. J. \& Lüdecke, J. (2001). J. Phys. Condens. Matter, 13, 9923-9936.
Smaalen, S. van, Shaz, M., Palatinus, L., Daniels, P., Galli, F., Nieuwenhuys, G. J. \& Mydosh, J. A. (2004). Phys. Rev. B, 69, 014103.

Spijkerman, A., de Boer, J. L., Meetsma, A., Wiegers, G. A. \& van Smaalen, S. (1997). Phys. Rev. B, 56, 13757-13767.

Spijkerman, A., Meetsma, A., de Boer, J. L., Gao, Y. \& van Smaalen, S. (1995). Phys. Rev. B, 52, 3892-3899.

Stöwe, K. \& Wagner, F. R. (1998). J. Solid State Chem. 138, 160-168.

Straub, T., Finteis, T., Claessen, R., Steiner, P., Hufner, S., Blaha, P., Oglesby, C. S. \& Bucher, E. (1999). Phys. Rev. Lett. 82, 4504-4507.

Sunshine, S. A. \& Ibers, J. A. (1986). Inorg. Chem. 25, 4355-4358.

Ter Haar, L. W., DiSalvo, F. J., Bair, H. E., Fleming, R. M., Waszczak, J. V. \& Hatfield, W. E. (1987). Phys. Rev. B, 35, 1932-1938.

Thomson, R. E., Burk, B., Zettl, A. \& Clarke, J. (1994). Phys. Rev. B, 49, 16899-16916.

Toledano, J. C. \& Toledano, P. (1987). The Landau Theory of Phase Transitions. Singapore: World Scientific.

Tsutsumi, K. (1982). Phys. Rev. B, 26, 5765-5759.

Tsutsumi, K., Takagaki, T., Yamamoto, M., Shiozaki, Y., Ido, M., Sambongi, T., Yamaya, K. \& Abe, Y. (1977). Phys. Rev. Lett. 39, $1675-1676$.

Voit, J. (1995). Rep. Prog. Phys. 58, 977-1116.

Vucic, Z., Meetsma, A. \& de Boer, J. L. (1993). J. Phys. IV (Paris) Colloque C2, 3, 129-132.

Wiegers, G. A., de Boer, J. L., Meetsma, A. \& van Smaalen, S. (2001). Z. Kristallogr. 216, 45-50.

Williams, J. M., Schultz, A. J., Underhill, A. E. \& Carneiro, K. (1983). In Extended Linear Chain Compounds, Vol. 1, edited by J. S. Miller. New York: Plenum Press.

Wilson, J. A. (1979). Phys. Rev. 19, 6456-6468.

Wilson, J. A., DiSalvo, F. J. \& Mahajan, S. (1975). Adv. Phys. 24, 117-201.

Wolff, P. M. de, Janssen, T. \& Janner, A. (1981). Acta Cryst. A37, 625-636.

Yamamoto, A., Onoda, M., Takayama-Muromachi, E., Izumi, F., Ishigaki, T. \& Asano, H. (1990). Phys. Rev. B, 42, 4228-4239. 